\title{
A Prognostic Model Based on Preoperative MRI Predicts Overall Survival in Patients with Diffuse Gliomas
}

A. Hilario, J.M. Sepulveda, A. Perez-Nuñez, E. Salvador, J.M. Millan, A. Hernandez-Lain, V. Rodriguez-Gonzalez, A. Lagares, and A. Ramos

\begin{abstract}
BACKGROUND AND PURPOSE: Diffuse gliomas are classified as grades II-IV on the basis of histologic features, with prognosis determined mainly by clinical factors and histologic grade supported by molecular markers. Our aim was to evaluate, in patients with diffuse gliomas, the relationship of relative CBV and ADC values to overall survival. In addition, we also propose a prognostic model based on preoperative MR imaging findings that predicts survival independent of histopathology.
\end{abstract}

MATERIALS AND METHODS: We conducted a retrospective analysis of the preoperative diffusion and perfusion MR imaging in 126 histologically confirmed diffuse gliomas. Median relative CBV and ADC values were selected for quantitative analysis. Survival univariate analysis was made by constructing survival curves by using the Kaplan-Meier method and comparing subgroups by log-rank probability tests. A Cox regression model was made for multivariate analysis.

RESULTS: The study included 126 diffuse gliomas (median follow-up of 14.5 months). ADC and relative CBV values had a significant influence on overall survival. Median overall survival for patients with $A D C<0.799 \times 10^{-3} \mathrm{~mm}^{2} / \mathrm{s}$ was $<1$ year. Multivariate analysis revealed that patient age, relative $C B V$, and $A D C$ values were associated with survival independent of pathology. The preoperative model provides greater ability to predict survival than that obtained by histologic grade alone.

CONCLUSIONS: ADC values had a better correlation with overall survival than relative CBV values. A preoperative prognostic model based on patient age, relative CBV, and ADC values predicted overall survival of patients with diffuse gliomas independent of pathology. This preoperative model provides a more accurate predictor of survival than histologic grade alone.

ABBREVIATION: $r C B V=$ relative cerebral blood volume

$D$ iffuse gliomas are the second most common primary CNS neoplasms, behind meningiomas, and account for roughly $80 \%$ of primary malignant brain tumors. ${ }^{1-3}$

Diffuse gliomas are a heterogeneous group of neoplasms classified according to the World Health Organization system as grades II-IV on the basis of histologic features, including cell density nuclear atypia, mitotic activity, endothelial proliferation, and

Received June 10, 2013; accepted after revision November 10.

From the Departments of Radiology (A.H., A.R., E.S., J.M.M.), Neuropathology (A.H.-L.), Medical Oncology (J.M.S.), Radiation Oncology (V.R.-G.), and Neurosurgery (A.P.-N., A.L.), Hospital 12 de Octubre, Madrid, Spain.

Paper previously presented at: Annual Meeting of the American Society of Neuroradiology, May 20-23, 2013; San Diego, California.

Level of contribution: A. Hilario, J.M. Sepulveda, A. Ramos, A. Perez-Nuñez, A. Lagares, and A. Hernandez-Lain contributed to the concept and design of the study, the analysis and interpretation of the results, and the writing of the article. All authors approved the final submitted version

Please address correspondence to A. Hilario, MD, Department of Radiology, Hospital 12 de Octubre, Avenida de Cordoba s/n 28041 Madrid, Spain; e-mail:

amayahilario@yahoo.es

http://dx.doi.org/10.3174/ajnr.A3837 necrosis. ${ }^{3,4}$ The prognosis for these tumors is determined mainly by histologic grade, ${ }^{3}$ with a median survival of approximately 3 years for anaplastic astrocytoma and 1 year for glioblastoma. ${ }^{5}$ However, it has been shown that histologic classification of gliomas remains insufficient due to its lack of precision in terms of prognosis. ${ }^{6}$ This classification for phenotype determination and grade is also subjective due to the fact that 1 histologic subtype could comprise different molecular subtypes with different prognoses. ${ }^{6}$ In recent years, extensive molecular studies ${ }^{7}$ have identified diagnostic and prognostic markers in gliomas that support the World Health Organization histologic classification. ${ }^{4} 1 \mathrm{p} 19 \mathrm{q}$ codeletion, methylation of the $\mathrm{O}^{6}$-methylguanine DNA methyltransferase gene promoter, and isocitrate dehydrogenases 1 and 2 gene mutations are the 3 most important markers in diffuse gliomas and may influence the sensitivity of these tumors to treatment. ${ }^{4}$

The role of neuroimaging has been widely studied in the literature, not only in the preoperative grading of diffuse gliomas ${ }^{8}$ but also in the determination of tumor response to treatment or tu- 
mor progression. ${ }^{9}$ However, the results about the utility of imaging techniques have been mixed, especially those in diffusion and perfusion MR imaging, in the determination of the prognosis of diffuse gliomas. The purposes of our study were as follows: 1) to evaluate the relationship of relative cerebral blood volume (rCBV) and ADC values to overall survival in a group of patients with histologically confirmed diffuse gliomas, and 2) to establish a prognostic model based on preoperative MR imaging findings that could predict overall survival regardless of histology.

\section{MATERIALS AND METHODS Study Population}

We conducted a retrospective analysis of the preoperative MR imaging findings of 126 diffuse gliomas, which were histologically confirmed by surgical resection or stereotactic biopsy.

The patients were selected from a larger group of 162 consecutive patients with diffuse gliomas admitted to our hospital during a 4-year period (2006-2010). Inclusion criteria were preoperative diffusion and perfusion $\mathrm{MR}$ imaging data suitable for evaluation and histologic diagnosis. Thirteen patients were excluded for incomplete follow-up (they did not continue at our institution). Another 23 patients were rejected because they underwent radiation therapy or chemotherapy before inclusion in the study. For histopathologic diagnosis, the samples were analyzed individually by 2 neuropathologists and were classified as low-grade (grade II) and high-grade (grades III and IV) according to the 2007 World Health Organization Classification of Tumors of the Central Nervous System. ${ }^{10}$ In all cases, patient treatment after histologic diagnosis was decided in the multidisciplinary neurooncology unit of our hospital on the basis of standard management (National Comprehensive Cancer Network guidelines for the treatment of the CNS cancers; www.nccn.org).

\section{Perfusion MR Imaging Protocol and Processing}

All patients were examined with the same imaging acquisition protocol on a 1.5T MR imaging system (Signa Excite; GE Healthcare, Milwaukee, Wisconsin) by using an 8-channel head coil. After an initial loading dose of 2-mL gadobutrol ${ }^{11}$ (Gadovist, 1 $\mathrm{mmol} / \mathrm{mL}$; Berlex Laboratories, Wayne, New Jersey), dynamic contrast-enhanced $\mathrm{T} 2{ }^{*}$-weighted perfusion MR imaging was performed by using a gradient-echo echo-planar imaging acquisition during the first pass of a bolus of gadobutrol. Nineteen sections were selected for perfusion MR imaging to cover the tumor on the basis of T2-weighted fast spin-echo $(\mathrm{TR}=4100 \mathrm{~ms}, \mathrm{TE}=85 \mathrm{~ms})$ or FLAIR (TR $=8000 \mathrm{~ms}$, $\mathrm{TE}=120 \mathrm{~ms}$, TI $=2000 \mathrm{~ms}$ ) images. Imaging parameters were as follows: $\mathrm{TR} / \mathrm{TE}=2000 / 21.1 \mathrm{~ms}$, FOV $=26 \times 26 \mathrm{~cm}$, section thickness $=4 \mathrm{~mm}$ with a $0.4-\mathrm{mm}$ skip, matrix $=128 \times 128$, flip angle $=90^{\circ}$. A series of 40 multisection acquisitions was acquired at 0.2 -second intervals; the total acquisition time was 1 minute 21 seconds. The first 8 acquisitions were performed before the contrast agent injection to establish a precontrast baseline. At the eighth acquisition, $0.1 \mathrm{mmol} / \mathrm{kg}$ of body weight of gadobutrol was injected with a power injector (Spectris Solaris EP; Medrad, Indianola, Pennsylvania) at a rate of $5 \mathrm{~mL} / \mathrm{s}$ through an 18-gauge intravenous catheter, immediately followed by a bolus injection of saline at the same rate for a total of $20 \mathrm{~mL}$.
The dynamic susceptibility-weighted contrast-enhanced MR perfusion images were processed on an Advantage Workstation by using FuncTool software (GE Healthcare). CBV maps were generated by calculating the change in relaxation rate $\left(\Delta \mathrm{R} 2^{*}\right)$ by using the following equation: $\Delta \mathrm{R} 2^{*}(\mathrm{t})=-\log$ neperien $\left(\mathrm{S}(\mathrm{t}) / \mathrm{S}_{0}\right) /$ time-echo, where $\mathrm{S}(\mathrm{t})$ is the signal intensity at time $\mathrm{t}$ and $\mathrm{S}_{0}$ is the baseline (precontrast) signal intensity. Because the CBV must be expressed relative to an internal reference, we normalized it by expressing ratios relative to values measured in the normal white matter of the contralateral lobe. We have referred to these relative values as rCBV. On the color-coded rCBV maps, 2 neuroradiologists ( 5 and 9 years of clinical experience in perfusion MR imaging) placed multiple ROIs to cover the entire tumor area, which was defined on the basis of T2/FLAIR or postgadolinium abnormality. The maximum rCBV value was therefore selected for quantitative analysis by consensus between the 2 neuroradiologists. The standardized region of interest was $2-3 \mathrm{~mm}^{2}$, which was used for most of the tumor and white matter measurements. Care was taken not to include large intra- or peritumoral vessels because these can confound perfusion measurements. This method has been demonstrated to provide the most optimal interobserver and intraobserver reproducibility. ${ }^{12}$

\section{Diffusion MR Imaging Protocol and Data Evaluation}

The diffusion-weighted imaging included an axial single-shot spin-echo echo-planar sequence with the following parameters: $\mathrm{TR} / \mathrm{TE}=8000 / 78.2 \mathrm{~ms}$, section thickness $=5 \mathrm{~mm}$, number of sections $=26$, acquisition matrix $=128 \times 128, \mathrm{FOV}=26 \times 26 \mathrm{~cm}$, b-values of 0 and $1000 \mathrm{~s} / \mathrm{mm}^{2}$, diffusion gradient encoding in 6 orthogonal directions, and a total scanning time of 1 minute 4 seconds.

Postprocessing of ADC maps was performed by using commercially available FuncTool software (Advantage Workstation). ADC measurements were obtained by placing multiple ROIs (area ranging from 1 to $2 \mathrm{~mm}^{2}$ ) over the whole tumor, which was defined on the basis of FLAIR/T2 or postgadolinium abnormality. By visual inspection, ROIs were placed on areas showing the lowest intratumoral $\mathrm{ADC}$, and then the minimum ADC value was selected for quantitative analysis by consensus between the 2 neuroradiologists. In comparison with susceptibility imaging, care was taken not to include intralesional blood products that would significantly decrease ADC values. ADC values were expressed as $10^{-3} \mathrm{~mm}^{2} / \mathrm{s}$.

\section{Statistical Analysis}

The end point of the study was overall survival, which was evaluated from the date of initial diagnosis to death or, for living patients, from diagnosis to the last available follow-up. Median maximum $\mathrm{rCBV}$ and minimum ADC values were selected as the cutoff values for quantitative analysis. Histology, rCBV, and ADC values were first evaluated univariately by constructing probability curves according to the Kaplan-Meier method and by comparing them with the log-rank test. A $P$ value $<.05$ indicated a statistically significant difference.

Radiologic variables achieving a $P$ value $<.05$ in the univariate analysis, together with age and histology, were subsequently introduced in a multivariable stepwise proportional hazards regression analysis (Cox model) to identify a set of features indepen- 
dently associated with survival. From the data obtained from multivariable analysis, we proposed a preoperative prognostic model based on patient age and MR imaging findings that could predict overall survival in diffuse gliomas.

Furthermore, the predictive power of survival models built with preoperative factors (age and MR imaging findings), postoperative factors (age, MR imaging findings, and histologic grade), and histologic grade alone was compared for obtaining Harrell C and Somers D parameters. ${ }^{13}$ These parameters were proposed as measures of the general predictive power of general regression models by Harrell et al. ${ }^{14}$ The Somers D is the probability that when 1 of 2 subjects is observed to survive another, the survivor has the lower of the 2 hazard ratios predicted by the model, rather than the survivor having the higher of the 2 predicted hazard ratios. The Harrell $\mathrm{C}$ is the probability that the survivor has the lower hazard ratio plus half the probability that the 2 subjects have equal hazard ratios. Univariate and multivariate analyses were both performed for the whole series $(n=126)$. All calculated $P$ values were 2 -sided. Statistical analysis was performed by using the Statistical Package for the Social Sciences, Version 12 software (IBM, Armonk, New York) and by using STATA 12 (StataCorp, College Station, Texas).

\section{RESULTS}

\section{Patient Population}

The study included 80 male and 46 female patients. Within this group of 126 diffuse gliomas, the median age at diagnosis was 53.4 years. Samples for histologic diagnosis were obtained by surgical resection in 84 patients $(66.7 \%)$ and by stereotactic biopsy in the remaining $33.3 \%(n=42)$. According to histologic analysis, our series comprised 26 low-grade $(20.6 \%)$ and 100 high-grade gliomas (79.4\%). We included 95 astrocytomas and 31 oligodendroglial tumors. The histopathologic diagnoses are presented in Table 1 . The median follow-up was 14.5 months (range, 0-76 months between January 2006 and February 2012, with a median follow-up of 36 months for living patients). Four patients died within the first 3 weeks after surgery as a result of cerebral edema $(n=3)$ and multiple organ dysfunction syndrome $(n=1)$. Tables 2 and 3 summarize the treatment of patients classified according to tumor grade, $\mathrm{rCBV}$, and $\mathrm{ADC}$ values.

\section{Univariate Survival Analysis}

The Kaplan-Meier overall survival curves for diffuse gliomas classified by tumor grade are shown in Fig 1. Histologic diagnosis had a significant influence on survival, with a median survival of 50 months for patients with grade III gliomas and 10 months (95\% CI, 6.87-13.12 months) for grade IV tumors. At the end of followup, patients with grade II gliomas had not reached median overall survival. Significant differences were observed among the 3 tumor grades $(P<.001)$.

Median maximum $\mathrm{rCBV}$ and minimum ADC values were 5.195 and $0.799 \times 10^{-3} \mathrm{~mm}^{2} / \mathrm{s}$, respectively. Relative cerebral blood volume correlated significantly with survival as shown in Fig 2: Median survival was 11 months (95\% CI, 7.543-14.457 months) for tumors with $\mathrm{rCBV} \geq 5.195$ and 50 months (95\% CI, 13.869-86.131 months) for tumors with perfusion values lower than the median maximum $\mathrm{rCBV}(P<.001)$. Kaplan-Meier estimates of survival indicated that patients with an $\mathrm{rCBV}<5.195$ had a significantly better overall survival.

The Kaplan-Meier overall survival curves for diffuse gliomas classified by ADC are shown in Fig 3. Apparent diffusion coefficient had a significant influence on survival, even better than rCBV. The group of gliomas with minimum ADC $<0.799 \times$ $10^{-3} \mathrm{~mm}^{2} / \mathrm{s}$ had a median survival time of 8 months $(95 \% \mathrm{CI}$, 5.194-10.806 months). At the end of the follow-up, patients with $\mathrm{ADC} \geq 0.799 \times 10^{-3} \mathrm{~mm}^{2} / \mathrm{s}$ had not reached median overall survival. Significant differences in overall survival were observed in diffuse gliomas classified by median minimum ADC $(P<$ .001 ). Our results also predicted that the median survival for diffuse gliomas with ADC values lower than $0.799 \times 10^{-3} \mathrm{~mm}^{2} / \mathrm{s}$ is $<12$ months. Table 4 summarizes the $P$ values for all variables evaluated by the univariate analysis.

As can be seen in Table 5, we also evaluated the median overall survival for the different histologic grades classified according to the rCBV and ADC values. In patients with low-grade tumors, ADC had a stronger correlation to survival than $\mathrm{rCBV}$.
Table 1: Histopathologic diagnosis distributed by tumor grade

\begin{tabular}{lcccr}
\hline \multicolumn{1}{c}{ Histology } & Grade II & Grade III & Grade IV & \multicolumn{1}{c}{ Total } \\
\hline Astrocytomas & $15(11.9 \%)$ & $7(5.6 \%)$ & $73(57.9 \%)(G B M)$ & $95(75.4 \%)$ \\
Oligodendrogliomas & $8(6.3 \%)$ & $10(7.9 \%)$ & & $18(14.2 \%)$ \\
Oligoastrocytomas & $3(2.4 \%)$ & $3(2.4 \%)$ & $7(5.6 \%)(G B M-O)$ & $13(10.4 \%)$ \\
Total & $26(20.6 \%)$ & $20(15.9 \%)$ & $80(63.5 \%)$ & $126(100 \%)$ \\
\hline
\end{tabular}

Note:-GBM indicates glioblastoma multiforme; GBM-O, glioblastoma with oligoastrocytoma grade IV.

Table 2: Treatment of patients classified by histologic grade, rCBV, and ADC values

\begin{tabular}{|c|c|c|c|c|c|}
\hline Histologic Grade & No Adjuvant Therapy & Radiotherapy & Chemotherapy (TMZ) & Chemoradiotherapy & Total \\
\hline Grade II & $7(5.6 \%)$ & $2(1.6 \%)$ & $14(11.2 \%)$ & $7(5.6 \%)$ & $24 \%$ \\
\hline Grade III & $1(0.8 \%)$ & $0(0 \%)$ & $6(4.8 \%)$ & $11(8.8 \%)$ & $14.4 \%$ \\
\hline Grade IV & $13(10.4 \%)$ & $0(0 \%)$ & $2(1.6 \%)$ & $63(49.6 \%)$ & $61.6 \%$ \\
\hline Total & $16.8 \%$ & $1.6 \%$ & $17.6 \%$ & $64 \%$ & $100 \%$ \\
\hline \multicolumn{6}{|l|}{ rCBV values } \\
\hline$r C B V \geq 5.195$ & $11(8.7 \%)$ & $0(0.0 \%)$ & $4(3.2 \%)$ & $48(38.1 \%)$ & $50 \%$ \\
\hline $\mathrm{rCBV}<5.195$ & $11(8.7 \%)$ & $2(1.6 \%)$ & $18(14.3 \%)$ & $32(25.4 \%)$ & $50 \%$ \\
\hline Total & $17.5 \%$ & $1.6 \%$ & $17.5 \%$ & $63.5 \%$ & $100 \%$ \\
\hline \multicolumn{6}{|l|}{$A D C$ values } \\
\hline $\mathrm{ADC}<0.799 \times 10^{-3} \mathrm{~mm}^{2} / \mathrm{s}$ & $13(10.3 \%)$ & $0(0.0 \%)$ & $3(2.3 \%)$ & $47(37.4 \%)$ & $50 \%$ \\
\hline$A D C \geq 0.799 \times 10^{-3} \mathrm{~mm}^{2} / \mathrm{s}$ & $8(6.4 \%)$ & $2(1.6 \%)$ & 19 (15\%) & $34(27 \%)$ & $50 \%$ \\
\hline Total & $16.7 \%$ & $1.6 \%$ & $17.3 \%$ & $64.4 \%$ & $100 \%$ \\
\hline
\end{tabular}

Note:-TMZ indicates temozolomide. 
Table 3: Salvage therapy classified by histologic grade, ADC and

\begin{tabular}{lcc}
\hline rCBV values & $\begin{array}{c}\text { Salvage } \\
\text { Surgery }\end{array}$ & $\begin{array}{c}\text { Additional } \\
\text { Chemotherapy }\end{array}$ \\
\hline Grade II & $6(4.8 \%)$ & $1(0.8 \%)$ \\
Grade III & $2(1.6 \%)$ & $2(1.6 \%)$ \\
Grade IV & $11(8.7 \%)$ & $15(12 \%)$ \\
Total & $19(15.1 \%)$ & $18(14.4 \%)$ \\
rCBV $\geq 5.195$ & $8(6.3 \%)$ & $11(8.7 \%)$ \\
rCBV $<5.195$ & $11(8.7 \%)$ & $7(5.7 \%)$ \\
Total & $19(15.1 \%)$ & $18(14.4 \%)$ \\
ADC $<0.799 \times 10^{-3} \mathrm{~mm}^{2} / \mathrm{s}$ & $10(8 \%)$ & $12(9.6 \%)$ \\
ADC $\geq 0.799 \times 10^{-3} \mathrm{~mm}^{2} / \mathrm{s}$ & $9(7.1 \%)$ & $6(4.8 \%)$ \\
Total & $19(15.1 \%)$ & $18(14.4 \%)$ \\
\hline
\end{tabular}

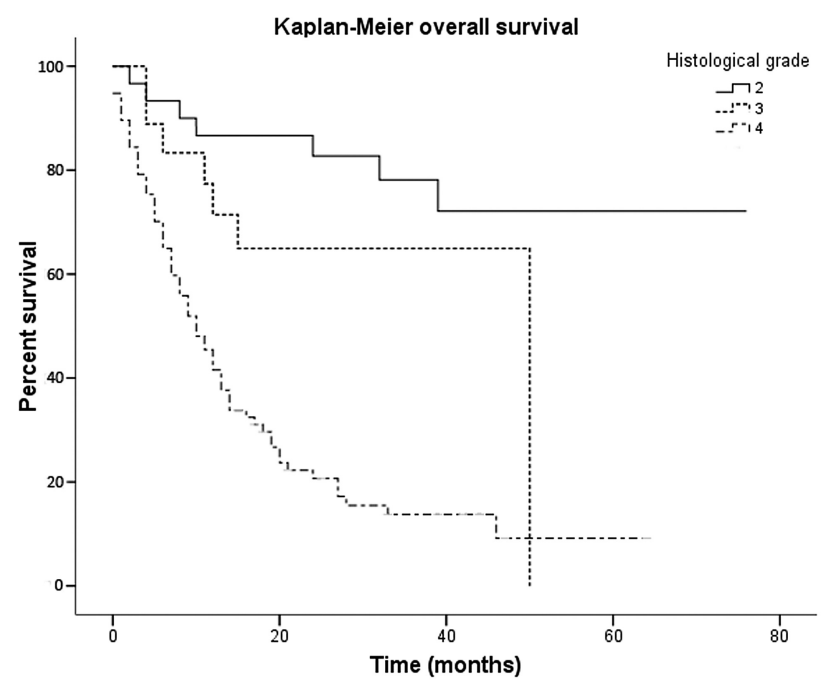

FIG 1. Kaplan-Meier survival curves of 126 diffuse gliomas show overall survival to be concordant with histologic grade. Low-grade gliomas had the longest overall survival, followed by grade III and IV tumors.

\section{Multivariate Survival Analysis}

We also combined the favorable imaging variables $(P<.05)$ with patient age and histology in a multivariate Cox regression analysis, which demonstrated that age, ADC, and $\mathrm{rCBV}$ values were independent predictors of survival. Determining imaging characteristics that may predict survival is a challenge in neuro-oncology. We can then set a preoperative prognostic model for diffuse gliomas based on ADC, rCBV, and patient age that predicts overall survival independent of pathology. Table 6 summarizes the multivariate associations of variables with survival in the whole series. Table 7 compares the predictive value of different possible multivariate Cox regression models built with pre- and postoperative factors. The preoperative model had a similar predictive power to that obtained postoperatively by using histologic information. Preoperative information presents greater predictive ability of survival than that obtained by histologic grade alone.

\section{DISCUSSION}

The management of diffuse gliomas has undergone many changes during the past 2 decades, with novel approaches to surgery, radiation, and chemotherapy improving survival and quality of life for patients. ${ }^{2}$ With regard to surgery, a more extensive surgical resection has been associated with longer life expectancy for both low- and high-grade newly diagnosed gliomas. ${ }^{15}$ On the other

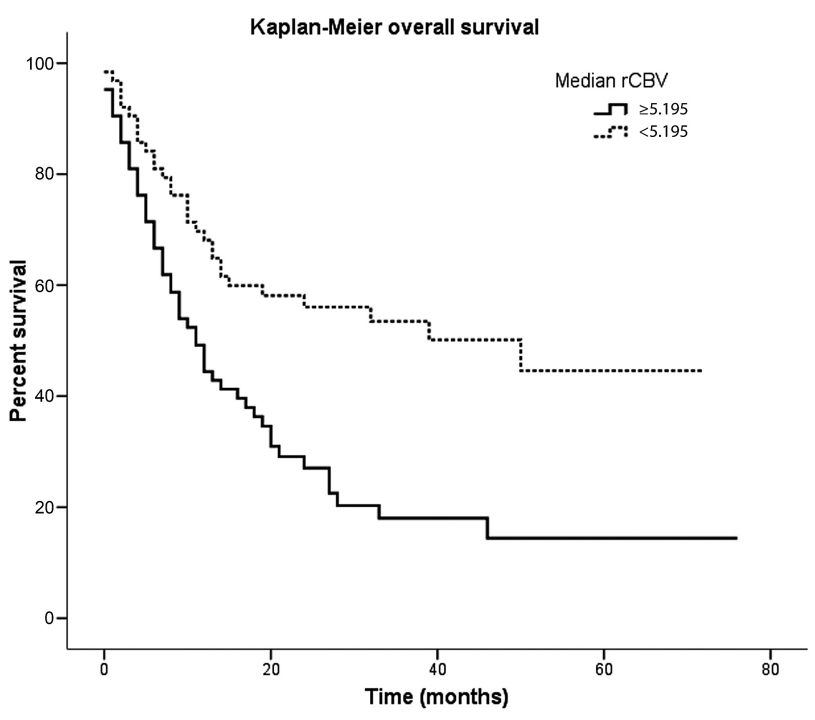

FIG 2. Kaplan-Meier survival curves of 126 patients with diffuse gliomas. Survival curves are plotted according to the classification based on median rCBV values. Relative cerebral blood volume has a significant influence on overall survival, with a median survival of 11 months for tumors with perfusion values lower than the median rCBV.

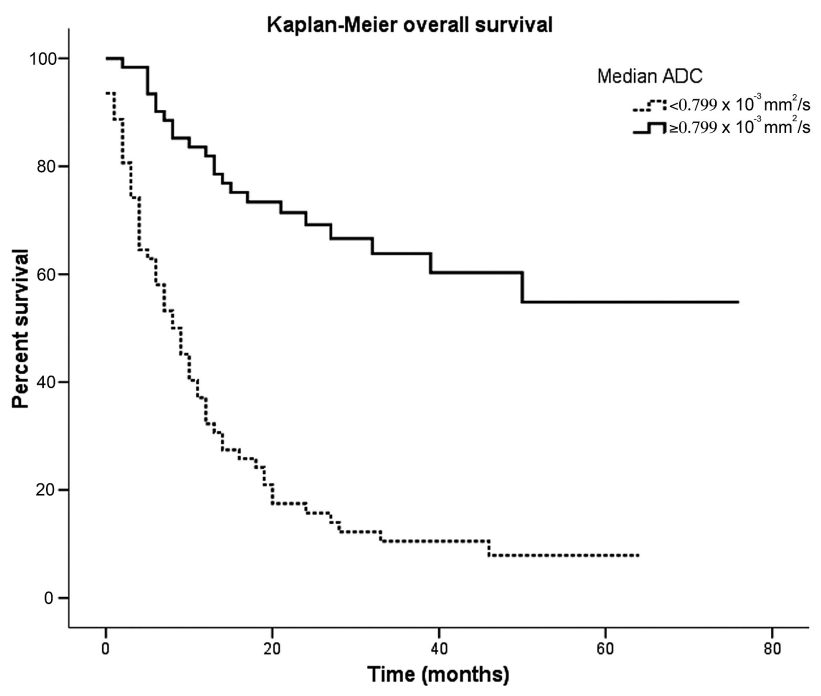

FIG 3. Overall survival calculated by using Kaplan-Meier curves for all patients stratified by median ADC values. Apparent diffusion coefficient has a significant influence on survival, even better than rCBV. In our series, the median survival for diffuse gliomas with $A D C$ values lower than $0.799 \times 10^{-3} \mathrm{~mm}^{2} / \mathrm{s}$ was $<12$ months.

hand, knowledge about the molecular biology of CNS gliomas has provided a dynamic classification of tumors that helps determine prognosis and the likelihood of a therapeutic response. ${ }^{2}$

The role of imaging techniques has also changed significantly during the past few years. First, several studies ${ }^{8,16}$ have demonstrated that $\mathrm{ADC}$ and $\mathrm{rCBV}$ values could serve as a noninvasive method of approximating tumor grade in diffuse gliomas. Second, researchers in several studies ${ }^{17,18}$ have proved the utility of rCBV in the prediction of median time to progression and propensity for malignant transformation. In view of the findings of these studies, we have gone a step further by evaluating the relationship between rCBV and ADC values and overall survival in a large group of 126 histologically confirmed diffuse gliomas. In this article, we have also 
attempted to create a preoperative prognostic model able to predict overall survival independent of pathology.

When selecting the rCBV and ADC cutoff values, we found discrepancies among previously published reports. Those differences found in rCBV values may be due to the use of a loading dose of contrast, the preload-postprocessing algorithm, and the use of sophisticated tools of segmentation instead of the regionof-interest-approach. ${ }^{19,20}$ Traditional region-of-interest measurements of rCBV remain the preferred technique for perfusion analysis in that they show the highest correlation to tumor grade. ${ }^{21}$ However, it has been demonstrated that the histogram method has an advantage over the region-of-interest-approach, an operator-dependent method, in the assessment of tumor response to treatment, especially with angiogenesis inhibitors. ${ }^{21}$ Law et $a^{18}$ used an rCBV value of 1.74 to predict median time to

Table 4: Summary of univariate associations with survival in the whole series

\begin{tabular}{lccc}
\hline & \multicolumn{3}{c}{ Univariate Analysis } \\
\cline { 2 - 4 } \multicolumn{1}{c}{ Prognostic Factor } & Hazard Ratio & $\mathbf{9 5 \%} \mathbf{C l}$ & $\boldsymbol{P}$ Value \\
\hline Patient age & 1.062 & $1.042-1.082$ & $<.001$ \\
rCBV median $\geq 5.195$ & 2.238 & $1.419-3.531$ & .001 \\
ADC median $<0.799 \times 10^{-3} \mathrm{~mm}^{2} / \mathrm{s}$ & 4.766 & $2.882-7.883$ & $<.0001$ \\
Histologic grade (ref grade II) & & & $<.0001$ \\
$\quad$ Grade III & 2.411 & $0.838-6.934$ & .0103 \\
$\quad$ Grade IV & 7.611 & $3.418-16.944$ & $<.0001$ \\
\hline
\end{tabular}

Note:-ref indicates reference.

Table 5: Median overall survival for histologic grades classified according to the rCBV and ADC values

\begin{tabular}{lccc}
\hline & No. (\%) & $\begin{array}{c}\text { Median Overall } \\
\text { Survival (mo) }\end{array}$ & $\mathbf{9 5 \% ~ C I}$ \\
\hline Grade II + ADC $<0.799 \times 10^{-3} \mathrm{~mm}^{2} / \mathrm{s}$ & $3(2.4)$ & 4 & $0.799-7.201$ \\
Grade II + ADC $\geq 0.799 \times 10^{-3} \mathrm{~mm}^{2} / \mathrm{s}$ & $23(18.2)$ & - & - \\
Grade III + ADC $<0.799 \times 10^{-3} \mathrm{~mm}^{2} / \mathrm{s}$ & $7(5.6)$ & 6 & $1.706-10.294$ \\
Grade III + ADC $\geq 0.799 \times 10^{-3} \mathrm{~mm}^{2} / \mathrm{s}$ & $13(10.3)$ & 50 & - \\
Grade IV + ADC $<0.799 \times 10^{-3} \mathrm{~mm}^{2} / \mathrm{s}$ & $58(46)$ & 9 & $6.307-11.693$ \\
Grade IV + ADC $\geq 0.799 \times 10^{-3} \mathrm{~mm}^{2} / \mathrm{s}$ & $22(17.5)$ & 17 & $6.152-27.848$ \\
Grade II + rCBV $\geq 5.195$ & $0(0)$ & - & - \\
Grade II + rCBV $<5.195$ & $26(20.6)$ & - & - \\
Grade III + rCBV $\geq 5.195$ & $5(4)$ & 11 & - \\
Grade III + rCBV $<5.195$ & $15(11.9)$ & 50 & - \\
Grade IV + rCBV $\geq 5.195$ & $59(46.8)$ & 10 & $6.301-13.699$ \\
Grade IV + rCBV $<5.195$ & $21(16.7)$ & 10 & $5.639-14.361$ \\
\hline
\end{tabular}

Note:- - indicates that at the end of follow-up, patients had not reached median overall survival.

Table 6: Summary of multivariate associations with survival in the whole series $(n=126)$

\begin{tabular}{lccc}
\hline & \multicolumn{3}{c}{ Multivariate Analysis } \\
\cline { 2 - 4 } \multicolumn{1}{c}{ Prognostic Factor } & Hazard Ratio & $\mathbf{9 5 \%} \mathrm{Cl}$ & $\boldsymbol{P}$ Value \\
\hline Patient age & 1.054 & $1.031-1.077$ & $<.001$ \\
rCBV median $\geq 5.195$ & 1.608 & $0.928-2.784$ & .090 \\
ADC median $<0.799 \times 10^{-3} \mathrm{~mm}^{2} / \mathrm{s}$ & 2.339 & $1.291-4.240$ & .005 \\
Histologic grade (ref grade II) & & & .027 \\
$\quad$ Grade III & 1.484 & $0.495-4.449$ & .481 \\
$\quad$ Grade IV & 3.538 & $1.328-9.427$ & .012 \\
\hline
\end{tabular}

Note:-ref indicates reference. progression in a group of 189 diffuse gliomas. On the other hand, Zacharaki et $\mathrm{al}^{5}$ performed a survival analysis of patients with high-grade gliomas based on mean rCBV and ADC values. For the MR imaging analysis, the threshold values selected in our study were median $\mathrm{rCBV}$ and ADC, similar to those used by Saraswathy et $\mathrm{al}^{22}$ for assessing MR imaging markers that predict survival in patients with glioblastoma before adjuvant therapy.

In our series, we have confirmed the relationship between histologic diagnosis and survival in diffuse gliomas. The median surliterature by Labussiere $\mathrm{al}^{4}$ and DeAngelis. ${ }^{23}$

We have also demonstrated that perfusion and diffusion imaging have a significant influence on the survival of patients with diffuse gliomas. The overall survival of those patients with tumors differed significantly depending on the $\mathrm{ADC}$ and $\mathrm{rCBV}$ values. Not only did the lower ADC and higher rCBV values correlate with a worse prognosis but the median overall survival for patients with ADC $<0.799 \times 10^{-3} \mathrm{~mm}^{2} / \mathrm{s}$ was $<1$ year.

However, the most important conclusion that can be drawn from our data is that minimum $\mathrm{ADC}$ and maximum rCBV values predict overall survival regardless of histologic grade. This finding represents an advance in the understanding of gliomas, postulating ADC and $\mathrm{rCBV}$ as potential biomarkers for survival.

The relationship of ADC to survival is based on the assumption that water diffusivity within the extracellular component is inversely related to the content and attenuation of the intracellular space. ${ }^{24}$ Quantitative information of the restriction of water molecule movement can be observed by calculating the ADC values from DWI. ${ }^{25}$ Thus, brain neoplasms with higher cellularity or with higher grades show a significant reduction in ADC values. ${ }^{26}$ These results support the findings, in a group of 39 highgrade gliomas, previously published by Hamstra et al, ${ }^{27}$ who reported that tumor assessment based on functional diffusion maps provides an early biomarker for response, time to progression, and overall survival in patients in malignant gliomas. Recently, Pope et $\mathrm{al}^{28,29}$ demon-

Table 7: Comparison of predictive performance of different Cox survival models built with prognostic factors obtained pre- and postoperatively

\begin{tabular}{|c|c|c|c|c|}
\hline Prognostic Models & Harrell C & $95 \% \mathrm{Cl}$ & Somers D & $95 \% \mathrm{Cl}$ \\
\hline Preoperative (age, $\mathrm{rCBV}$ median $\geq 5.195$, ADC median $<0.799 \times 10^{-3} \mathrm{~mm}^{2} / \mathrm{s}$ ) & 0.77 & $0.71-0.83$ & 0.61 & $0.45-0.77$ \\
\hline $\begin{array}{l}\text { Postoperative (age, rCBV median } \geq 5.195 \text {, ADC median }<0.799 \times 10^{-3} \mathrm{~mm}^{2} / \mathrm{s} \text {, } \\
\text { histologic grade) }\end{array}$ & 0.78 & $0.72-0.84$ & 0.64 & $0.48-0.81$ \\
\hline Histologic grade & 0.67 & $0.61-0.73$ & 0.35 & $0.22-0.48$ \\
\hline
\end{tabular}


strated the relevance of ADC values in the management of gliomas. These authors showed that pretreatment ADC histogram analysis could stratify progression-free and overall survival in patients with newly diagnosed and recurrent glioblastomas treated with bevacizumab. Regarding these articles, ${ }^{27-29}$ our work contributes, as an innovation, the inclusion of low- and high-grade tumors in evaluating the relationship between ADC values and overall survival.

The association of rCBV with survival is based on the theory that vascular proliferation defines the biologic aggressiveness of gliomas and is considered a very important factor in histopathologic grading. ${ }^{24,30} \mathrm{CBV}$ measurements correlate with tumor grade and histologic findings of increased tumor vascularity. Because higher vascularity corresponds to a higher tumor grade, as the grade of astrocytoma increases, the maximal tumor CBV tends to increase. ${ }^{24,31}$ Law et al ${ }^{18}$ demonstrated in 2008 that dynamic susceptibility-weighted contrast-enhanced MR perfusion imaging could be used to predict median time to progression in patients with gliomas, independent of pathology. However, in that series, it was found that $\mathrm{rCBV}$ was not significantly associated with overall survival $(P>.63)$. Our results differ from those published by Law et al, ${ }^{18}$ demonstrating that rCBV has a significant influence on overall survival of patients with gliomas. Both series comprised a large number of patients (126 versus 189), but the reason for the opposite results may be the inclusion of a greater percentage of grade II tumors in the series of Law et al. ${ }^{18}$ Our results are also in disagreement with those published by Zacharaki et al, ${ }^{5}$ who reported that region of interest-based characteristics in $\mathrm{rCBV}$ were not among the top-ranked variables for predicting survival in high-grade gliomas. An explanation for the differing results could be differences in the study population. Zacharaki et al included only high-grade tumors, which have generally increased rCBV on perfusion MR imaging.

As seen above, ADC and rCBV values may be considered MR imaging markers that predict survival in diffuse gliomas, which may be important for stratifying patients to different treatment protocols. For this reason, one of the goals of our study was the design of a preoperative model able to predict prognosis in diffuse gliomas. Zacharaki et $\mathrm{al}^{5}$ reported recently that prediction models based on machine-learning algorithms provide a more accurate predictor of prognosis in malignant gliomas than does histopathologic classification alone. Our research is similar to that of Zacharaki et al and presented, in a larger series (126 versus 67), the design of a more simple prognostic model based on patient age, $\mathrm{ADC}$, and $\mathrm{rCBV}$ values capable of predicting survival in diffuse gliomas independent of pathology. We have also demonstrated that the preoperative model provides greater predictive ability of survival than that obtained by histologic grade alone.

There are some limitations to our study. First, the major one is that it was performed retrospectively in a heterogeneous group that included astrocytomas and oligodendroglial tumors of any grade. A second potential limitation was the possible subjectivity and reproducibility of a region of interest-based technique for rCBV and ADC measurements. Finally, it is likely that improvement in the current World Health Organization classification with incorporation of molecular markers, as well as imaging bio- markers of outcome, will predict future understanding of glioma biology and therapy.

\section{CONCLUSIONS}

ADC values had a significant influence on overall survival, even better than $\mathrm{rCBV}$, with a median time of 8 months for the group of gliomas with $\mathrm{ADC}<0.799 \times 10^{-3} \mathrm{~mm}^{2} / \mathrm{s}$. A preoperative prognostic model based on patient age, $\mathrm{rCBV}$, and ADC values predicts overall survival of diffuse gliomas independent of pathology. This preoperative model provides greater predictive ability of survival than does histologic grade alone.

Disclosures: Ángel Pérez-Nuñez—RELATED: Fondo de Investigación Sanitaria, Instituto de Salud Carlos III, ${ }^{*}$ Comments: Some of the data of this work are, in part, shared with another investigation, for which we received financial support. The title of that project was "Estudio Radiológico, Histológico y Molecular de la Angiogénesis Tumoral en Gliomas Mediante Biopsia Selectiva" ("Radiological, Histological and Molecular Study of Tumoral Angiogenesis in Gliomas, by Means of Selective Biopsy"). Ana Ramos-UNRELATED: Grants/Grants Pending: Fondo de Investigación Sanitaria. * *Money paid to the institution.

\section{REFERENCES}

1. Bondy ML, Scheurer ME, Malmer B, et al. Brain tumor epidemiology: consensus from the brain tumor epidemiology consortium (BTEC). Cancer 2008;113:1953-68

2. Jansen M, Yip S, Louis DN. Molecular pathology in adult gliomas: diagnostic, prognostic and predictive markers. Lancet Neurol 2010;9:717-26

3. Theeler BJ, Yung WK, Fuller GN, et al. Moving toward molecular classification of diffuse gliomas in adults. Neurology 2012;79: 1917-26

4. Labussiere M, Wang XW, Idbaih A, et al. Prognostic markers in gliomas. Future Oncol 2010;6:733-39

5. Zacharaki EI, Morita N, Bhatt P, et al. Survival analysis of patients with high-grade gliomas based on data mining of imaging variables. AJNR Am J Neuroradiol 2012;33:1065-71

6. Ducray F, El Hallani S, Idbaih A. Diagnostic and prognostic markers in gliomas. Curr Opin Oncol 2009;21:537-42

7. Yan $\mathrm{H}$, Parsons DW, Jin G, et al. IDH1 and IDH2 mutations in gliomas. N Engl J Med 2009;360:765-73

8. Hilario A, Ramos A, Perez-Nuñez A, et al. The added value of apparent diffusion coefficient to cerebral blood volume in the preoperative grading of diffuse gliomas. AJNR Am J Neuroradiol 2012;33:701-07

9. Caroline I, Rosenthal MA. Imaging modalities in high-grade gliomas: pseudoprogression, recurrence, or necrosis? J Clin Neurosci 2012;19:633-37

10. Louis DN, Ohgaki H, Wiestler OD, et al, eds. 2007 WHO Classification of Tumours of the Central Nervous System. 4th ed. Lyon, France: IARC Press; 2007

11. Lacerda S, Law M. Magnetic resonance perfusion and permeability imaging in brain tumors. Neuroimaging Clin N Am 2009;19:527-57

12. Wetzel SG, Cha S, Johnson G, et al. Relative cerebral blood volume measurements in intracranial mass lesions: interobserver and intraobserver reproducibility study. Radiology 2002;224:797-803

13. Newson R. Confidence intervals for rank statistics: Somers's D and extensions. Stata J 2006;6:308-34

14. Harrell FE Jr, Lee KL, Mark DB. Multivariate prognostic models: issues in developing models, evaluating assumptions and adequacy, and measuring and reducing errors. Stat Med 1996;15:361-87

15. Hardesty DA, Sanai N. The value of glioma extent of resection in the modern neurosurgical era. Front Neurol 2012;3:140-47

16. Law M, Yang S, Babb JS, et al. Comparison of cerebral blood volume and vascular permeability from dynamic susceptibility contrastenhanced perfusion MR imaging with glioma grade. AJNR Am J Neuroradiol 2004;25:746-55

17. Law M, Oh S, Babb JS, et al. Low-grade gliomas: dynamic suscepti- 
bility-weighted contrast-enhanced perfusion MR imaging: prediction of patient clinical response. Radiology 2006;238:658-67

18. Law M, Young RJ, Babb JA, et al. Gliomas: predicting time to progression or survival with cerebral blood volume measurements at dynamic susceptibility-weighted contrast-enhanced perfusion MR imaging. Radiology 2008;247:490-98

19. Boxerman JL, Prah DE, Paulson ES, et al. The role of preload and leakage correction in gadolinium-based cerebral blood volume estimation determined by comparison with MION as a criterion standard. AJNR Am J Neuroradiol 2012;33:1081-87

20. Paulson ES, Schmainda KM. Comparison of dynamic susceptibilityweighted contrast-enhanced MR methods: recommendations for measuring relative cerebral blood volume in brain tumors. Radiology 2008;249:601-13

21. Law M, Young R, Babb J, et al. Histogram analysis versus region of interest analysis of dynamic susceptibility contrast perfusion $M R$ imaging data in the grading of cerebral gliomas. AJNR Am J Neuroradiol 2007;28:761-66

22. Saraswathy S, Crawford FW, Lamborn KR, et al. Evaluation of MR markers that predict survival in patients with newly diagnosed GBM prior to adjuvant therapy. J Neurooncol 2009;91:69-81

23. DeAngelis LM. Brain tumors. N Engl J Med 2001;344:114-23

24. Cha S. Update on brain tumor imaging: from anatomy to physiology. AJNR Am J Neuroradiol 2006;27:475-87

25. Kono K, Inoue $\mathrm{Y}$, Nakayama $\mathrm{K}$, et al. The role of diffusion-weighted imaging in patients with brain tumors. AJNR Am J Neuroradiol 2001;22:1081-88

26. Sugahara T, Korogi $\mathrm{Y}$, Kochi M, et al. Usefulness of diffusionweighted MRI with echo-planar technique in the evaluation of cellularity in gliomas. J Magn Reson Imaging 1999;9:53-60

27. Hamstra DA, Chenevert TL, Moffat BA, et al. Evaluation of the functional diffusion map as an early biomarker of time-to-progression and overall survival in high-grade glioma. Proc Natl Acad Sci U S A 2005;102:16759-64

28. Pope WB, Lai A, Mehta R, et al. Apparent diffusion coefficient histogram analysis stratifies progression-free survival in newly diagnosed bevacizumab-treated glioblastoma. AJNR Am J Neuroradiol 2011;32:882-89

29. Pope WB, Qiao XJ, Kim HJ, et al. Apparent diffusion coefficient histogram analysis stratifies progression-free and overall survival in patients with recurrent GBM treated with bevacizumab: a multicenter study. J Neurooncol 2012;108:491-98

30. Hakyemez B, Erdogan C, Ercan I, et al. High-grade and low- grade gliomas: differentiation by using perfusion MR imaging. Clin Radiol 2005;60:493-502

31. Sadeghi N, D'Haene N, Decaestecker C, et al. Apparent diffusion coefficient and cerebral blood volume in brain gliomas: relation to tumor cell density and tumor microvessel density based on stereotactic biopsies. AJNR Am J Neuroradiol 2008;29:476-82 